\title{
Does the Vigilance-Avoidance Gazing Behavior of Children with Separation Anxiety Disorder Change after Cognitive-Behavioral Therapy?
}

\author{
Tina In-Albon • Silvia Schneider
}

Published online: 31 March 2012

(C) Springer Science+Business Media, LLC 2012

\begin{abstract}
Cognitive biases are of interest in understanding the development of anxiety disorders. They also play a significant role during psychotherapy, where cognitive biases are modified in order to break the vicious cycle responsible for maintaining anxiety disorders. In a previous study, the vigilance-avoidance pattern was shown in children with separation anxiety disorder (In-Albon et al. Journal of Abnormal Child Psychology 38:225-235, 2010). The exhibited avoidance pattern may be essential for the maintenance of the anxiety disorder. Therefore, in the present study we used eye tracking methodology presenting disorder specific pictures to examine possible changes in the vigilance-avoidance pattern in 18 children with separation anxiety disorder after cognitive-behavioral treatment (CBT) and 13 healthy controls. Results indicated that following treatment, the vigilance pattern of children with separation anxiety disorder reduced significantly. Thus, the vigilanceavoidance pattern can be modified by CBT.
\end{abstract}

Keywords Attentional bias - Eye movement - Separation anxiety disorder · Vigilance-avoidance model · Cognitive behavioral therapy Eye tracking

The defining feature of separation anxiety disorder (SAD) is an excessive and unrealistic fear of separation from an attachment figure, beyond of what would be expected of a child's

\footnotetext{
T. In-Albon $(\bowtie)$

Department of Clinical Psychology and Psychotherapy,

Institute of Psychology, University of Basel,

Missionsstrasse 60/62,

CH-4055 Basel, Switzerland

e-mail: tina.in-albon@unibas.ch

S. Schneider

Clinical Child and Adolescent Psychology,

Ruhr-Universität Bochum,

Bochum, Germany
}

developmental level. The most frequently reported symptoms are separation related distress, avoidance of being alone or without an adult, and sleeping away from home or without a caregiver (Allen et al. 2010). A comprehensive review by Cartwright-Hatton et al. (2006) indicated a prevalence rate of almost $4 \%$ for SAD. Furthermore, SAD displays an early median age of onset at 7 years of age, and is therefore one of the earliest mental disorders in childhood (Kessler et al. 2005). Shear et al. (2006) showed that $36 \%$ of the respondents who were classified retrospectively with childhood SAD had an illness that persisted into adulthood. Moreover, SAD is a significant risk factor for the development of various mental disorders in adulthood (Brückl et al. 2007; Lewinsohn et al. 2008). Consequently, the importance of treatment for children with SAD is apparent. To improve current treatment of anxiety disorders and to develop more effective treatment strategies (In-Albon and Schneider 2007), it is crucial to promote knowledge of the risk and maintenance factors as well as of the mechanisms underlying treatment response.

According to cognitive theories, biases in information processing play an important role in the etiology and maintenance of anxiety disorders (e.g., Hadwin and Field 2010; Field 2006; MacLeod et al. 2002). In relation to anxiety, biases of selective attention are among the most extensively studied information-processing biases in adults and children (overview see Puliafico and Kendall 2006; Cisler and Koster 2010). Subsequent to cognitive models, cognitivemotivational models of anxiety have proposed an alternative pattern of attentional bias in anxiety (Mathews and Mackintosh 1998; Mogg and Bradley 1998). The two-stage, vigilanceavoidance hypothesis (Mogg and Bradley 1998) suggests that if the threat cue is highly aversive, attention is likely to be initially allocated towards it (vigilance hypothesis; Beck et al. 1985), but may subsequently be diverted away from it (avoidance hypothesis; Foa and Kozak 1986), in an attempt to reduce subjective discomfort elicited by the threat or to reduce the degree of danger of the situation. Assessing the vigilance- 
avoidance model requires a continuous measure of attention over time, such as the eye tracking methodology. Such methodology can assess the entire course of attention in the presence of threatening stimuli, (Bögels and Mansell 2004). Eye tracking research with anxious adults tends to support the vigilance-avoidance model (e.g., Garner et al. 2006; Rinck and Becker 2006). However, so far only three published eye tracking studies investigated the vigilance-avoidance model with anxious children, showing inconsistent results (Gamble and Rapee 2009; Heinrichs and Reinhold 2010; In-Albon et al. 2010). Gamble and Rapee (2009) investigated 43 children aged 7-17 (19 children aged $7-11)$ with different anxiety disorders and 49 controls (20 children aged 7-11). Stimuli consisted of facial expressions depicting negative, positive, and neutral emotions in adults. There were two eye tracking tasks, in which face pairs were presented for 500 and 3,000 ms, respectively. A naturalistic free-viewing condition without an ongoing task was employed. Their results were only partially consistent with the vigilance-avoidance hypothesis. When face pairs were presented for $500 \mathrm{~ms}$, anxious children oriented themselves away from happy faces but not from negative faces compared to non-anxious children. To investigate the time course of attentional deployment, the entire $3,000 \mathrm{~ms}$ were analyzed, divided into $1 \mathrm{~s}$ intervals. Thereby, all participants displayed a vigilance-avoidance pattern of attention, regardless of their anxiety level. The pilot study of Heinrichs and Reinhold with 9 low socially anxious children and 8 high socially anxious children used facial expressions depicting angry, happy and neutral emotions in adults and children. The pairs of pictures were presented for $4,000 \mathrm{~ms}$, which were divided into $500 \mathrm{~ms}$ intervals for the analyses. Results indicated that initially all children were more likely to attend toward the angry facial expressions with a vigilance reaction. However, the low socially anxious children adjusted then their attention to the threat, while the high socially anxious children showed a brief period of avoidance after the initial vigilance reaction then followed again by a vigilance reaction, similar to the initial vigilance reaction. In our own antecedent study (In-Albon et al. 2010) of the present one, we investigated disorder-specific children with SAD and nonanxious controls using disorder-specific stimuli. The results indicated that the vigilance-avoidance model could be shown in children with SAD. After an initial orienting period, children with SAD gazed significantly more at separating (threatening) pictures than non-anxious children. After three seconds the pattern reversed: children with SAD gazed significantly less at the separating pictures than non-anxious children. It is difficult to compare these three studies, since the samples (children with different anxiety disorders, non clinical children with social anxiety, children with SAD), the stimuli (facial expressions of adults and children and disorder-related pictures to SAD), and the duration of the stimulus presentation ( $3 \mathrm{~s}$ and $4 \mathrm{~s}$ ) diverged from another.

Cognitive biases are not only of interest in understanding the development of anxiety disorders, but also play a significant role during psychotherapy, where the biases are modified to break the vicious cycle responsible for maintaining anxiety disorders. The avoidance pattern may be essential for the maintenance of an anxiety disorder. Avoidance maintains anxiety, as it prevents the individual from evaluating vague stimuli sufficiently to perceive that they are not threatening, and from remaining exposed to threatening stimuli long enough to extinguish or habituate to their fear-arousing properties. The effects of treatment on attentional bias have been examined in adults in a variety of anxiety disorders. The results indicate that treatment can eliminate such biases for example in spider phobia, OCD, GAD, and social phobia (e.g., Mathews et al. 1995; Mattia et al. 1993; Mogg et al. 1995). Waters et al. (2008) investigated treatment effects on the interpretation and attentional bias in children with anxiety disorders. Following CBT, children with anxiety disorders showed significant reduced interpretation bias. However, there was no treatment effect on attentional bias toward threat pictures on a visual-probe task. The study did not include disorder specific stimuli material, which could have been insensitive to changes. Since the study investigated children with various anxiety disorders, disorder specific effects could not be examined. In a sample of 91 children with anxiety disorders, Legerstee et al. (2010) examined whether treatment response to stepped-care CBT is related to changes in selective attention. Results demonstrated that changes in selective attention to severe threat showed significant association with treatment success. However, both studies investigated different anxiety disorders with no disorder specific stimuli and most importantly, no continuous measure of attention was used.

Therefore, the present study, following up on our initial study (In-Albon et al. 2010), investigated gazing behavior of children with SAD diagnoses after receiving CBT. We compared whether their vigilance-avoidance viewing behavior changed after treatment and in comparison to a non-anxious control group. To the best of our knowledge, ours is the first study investigating the effects of CBT on attentional bias in children with anxiety disorders using a continuous measure of attention, such as eye tracking. In line with the results from adult studies of treatment effects on attention bias, we hypothesized that after receiving CBT, the pattern of the vigilance and the avoidance viewing behavior in children with $\mathrm{SAD}$ will change so that the SAD children will no longer show a vigilance peak which is followed by an avoidance viewing behavior. 


\section{Method}

Participants

The children in the present study had previously participated in the In-Albon et al. (2010) study. Participants were 18 children with a primary DSM-IV-TR diagnosis of SAD (10 girls and 8 boys) at pretreatment and 13 nonanxious control children (6 girls and 7 boys). The mean age of the children with $\mathrm{SAD}$ was 10.44 years $(\mathrm{SD}=1.09$, Range 8-13) and of the non-anxious control children 10.46 ( $\mathrm{SD}=1.61$, Range 8-13). The groups were comparable with respect to age $(t(29)=0.4, p=0.97)$ and gender $\left(\chi^{2}(1, N=31)=0.27, p=0.61\right)$. The sample size provided $77 \%$ power to detect a medium effect size $(f=0.25)$. Although we attempted to contact all previous participants, 9 children out of 40 (4 healthy controls and 5 children with SAD) could not be examined at the follow-up assessment because of technical difficulties or because their families chose to conduct the diagnostic follow-up assessment over the phone due to time issues. All children were diagnosed pre- and post-treatment using the Diagnostic Interview for Mental Disorders in Children and Adolescents (Kinder-DIPS; Schneider et al. 2009a), a structured interview in German based on DSM-IV-TR-criteria (American Psychiatric Association 2000). The children with SAD were participants in a cognitive-behavioral treatment study of SAD at the University of Basel, Switzerland. The children of the non-anxious control group were assessed with the Kinder-DIPS and had not experienced any mental disorders. They were paid for participation.

\section{Procedure}

Children and their parents gave written consent to participate in the separation anxiety disorder research project, approved by the Ethics Committee of Basel, Switzerland, which informed them of the child's right to withdraw at any time. None of the children withdrew their participation. Children were tested individually in a quiet room with the assistance of a graduate student.

\section{Treatment Program}

A treatment manual was developed for the purpose of the treatment study. The treatment condition included a baseline assessment, sixteen sessions of disorder-specific CBT for SAD including psychoeducation, cognitive restructuring and exposure in vivo (Schneider and Lavallee 2012; Schneider et al. 2011), as well as a follow-up assessment at 4-weeks (Schneider et al. 2011). The data collection for the 12 and 24-month follow-ups are ongoing.
Therapists were trained and supervised weekly by the last author. Each session was videotaped, with consent, for the purpose of analyzing treatment integrity. Videos from 10 randomly selected participants were each coded by two trained master students, using a standard coding checklist. Intraclass correlation coefficients indicated that ratings were highly consistent for adherence to the treatment protocol $(0.86, p<0.05)$.

\section{Measures}

Diagnoses of Mental Disorders To examine the children's current DSM-IV-TR diagnoses, we conducted a structured interview with each child and, separately, with each child's parents (i.e., either the mother or father or both together). Children's mental disorders were diagnosed using a structured interview, the Diagnostic Interview for Mental Disorders in Children and Adolescents (Kinder-DIPS; Schneider et al. 2009a, b), which has alternate forms for the child and the parent. The structured interview assesses all anxiety disorders, depression, attentiondeficit hyperactivity disorder, oppositional defiant disorder, sleep disorders, eating disorders, and elimination disorders. Reliabilities were calculated within a larger sample, overlapping with the present sample. The Kinder-DIPS had good validity and reliability for anxiety disorders (child version: kappa $=0.88$; parent version: $k a p p a=0.85$ ) and other axis I disorders (child version, kappa $=0.48$ 0.88 , parent version, kappa $=0.85-0.94$; Schneider et al. 2009b). Diagnoses were based on composite information from separate child and parent interviews. Doctoral students in clinical child psychology were first systematically trained in conducting the interviews. They were unaware of the child's original treatment condition. Two different interviewers conducted the parent and the child interviews. A diagnosis was given if either interviewer rendered the diagnosis.

Separation Anxiety, Child Rating The children completed the Separation Anxiety Inventory for children (SAI-C; Schneider and In-Albon 2005; In-Albon et al. 2011), a questionnaire consisting of 12 items assessing the degree to which the rater avoids 12 different separation situations using a 5-point scale ranging from 0 (never) to 4 (always). The stem of each item is "Because I am anxious, I'm avoiding... e.g., going to school, sleeping in my own bed". The psychometric properties of the SAI-C were good, including internal consistency (alpha $=0.71)$, test-retest reliability $(r=0.77)$, sensitivity to treatment change $(d=0.98)$, and construct validity covering all the major features commonly used to define separation anxiety disorder (In-Albon et al. 2011). Psychometric properties were assessed in a larger sample, overlapping with the present sample. 
Separation Anxiety, Parent Rating The parents rated the Separation Anxiety Inventory for parents (SAI-P; Schneider and In-Albon 2005; In-Albon et al. 2011), which assesses the parent's view of the degree to which the child avoids the same 12 separation situations as in the child's version. The psychometric properties of the SAI-P were good, including internal consistency (alpha $=0.71$ ), testretest reliability $(r=0.92)$, sensitivity to treatment change $(d=1.31)$, and construct validity covering all the major features commonly used to define separation anxiety disorder (In-Albon et al. 2011).

\section{Assessment of Vigilance and Avoidance}

The stimulus materials, eye tracking apparatus, and the eye tracking procedure were as described by In-Albon et al. (2010). Color photographs representing separating situations and reuniting situations were created. Separating photographs depicted a child and a woman, apparently the child's mother, separating from one another (e.g., by the woman leaving home or driving away in a car). Reuniting pictures depicted the child and woman greeting and embracing one another. The child in the photograph was either a boy or a girl, to allow matching the gender of the child in the photograph to the gender of the participant. Children in the pictures were between 8 and 10 years of age, a comparable age with the children investigated in this study. Before and after treatment, the same color photographs representing separating situations and reuniting situations were shown in a different randomization. Before receiving treatment children with SAD and non-anxious children rated the valence and arousal of the stimuli, indicating that children with SAD rated separating pictures as significantly more unpleasant, $t(37)=2.88, p<0.01$ and more arousing, $t(37)=3.08, p<$ 0.01 than did non-anxious children.

Eye Tracking Apparatus We tracked participants' gaze direction with the Tobii 1750 table-mounted eye tracker (Tobii Technology AB, Sweden). Each stimulus slide containing a pair of photographs was displayed on the computer screen while data recording participants' gaze direction were collected for both eyes simultaneously on an average of every $20 \mathrm{~ms}$ (i.e., $50 \mathrm{~Hz}$ ) and at an accuracy of less than $0.5^{\circ}$ (bias error). The eye tracking data were examined in terms of fixations recorded within these pictures (areas of interest). Mean latencies for each picture type were calculated after excluding latencies exceeding 3 SD above each participant's mean.

Eye Tracking Procedure The procedure was the same as in the In-Albon et al. (2010) study. Participants were seated approximately $60 \mathrm{~cm}$ away from the computer monitor. The instruction given to the child was: "Your task will be to look at the pictures on the screen until they disappear; move your eyes, but not your head". An experimenter who was unaware of whether the child had SAD, sat in the room with the child to ensure that the child paid attention to the screen, and that the eye tracker was working properly. After calibration, a single picture pair was shown for $4 \mathrm{~s}$. Prior to each picture display, a central fixation cross was shown for $1,000 \mathrm{~ms}$ in order to catch the participants' attention. The intertrial interval varied randomly within each block of trials between $750 \mathrm{~ms}$ and $1,250 \mathrm{~ms}$. The pairs of pictures were presented in counter balanced order across trails, one on the left side and the other on the right side of the screen. Each child underwent 9 blocks of trials, with each block containing 8 trials (i.e., eight 4-s presentations of a picture pair). The extent of each child's attention to threat was calculated as the percent of time the child spent looking at separating pictures as a function of total time he or she spent looking at either the separating or the reuniting picture.

\section{Results}

\section{Treatment Effects}

Of the 18 children with SAD receiving CBT treatment, all except one child achieved non-clinical status on the Kinder-DIPS at post-treatment. Presented in Table 1 are the mean scores of children with SAD and non-anxious children on the separation anxiety inventory, child- and parent version. Analysis of SAI-C revealed significant main effects of Time, $F(1,28)=21.18, p<0.001$, reflecting higher scores at pre-compared with post-treatment, and Group, $F(1,28)=23.18, p<0.01$, reflecting higher scores in the SAD compared with the non-anxious children. The interaction between Time and Group was also significant, $F(1,28)=9.11, p=0.005$, indicating greater difference between pre- and post-test anxiety scores in the anxious group than in the non-anxious group. Yet, the scores for the SAD group at post-treatment were significantly higher than for the non-anxious group $(p<0.01)$.

Table 1 Mean scores on the separation anxiety inventory of children with SAD and of non-anxious children at pre-and post-treatment

\begin{tabular}{llllll}
\hline & \multicolumn{2}{l}{ Pre-treatment } & & & \multicolumn{2}{l}{ Post-treatment } \\
\cline { 2 - 3 } \cline { 5 - 6 } \cline { 5 - 6 } & SAD & Non-anxious & & SAD & Non-anxious \\
& M (SD) & M (SD) & & M (SD) & M (SD) \\
\hline SAI-C & $2.00(0.75)$ & $0.58(0.66)$ & & $1.39(1.00)$ & $0.39(0.39)$ \\
SAI-P & $2.29(0.85)$ & $0.64(0.43)$ & & $1.09(0.71)$ & $0.42(35)$ \\
\hline
\end{tabular}

SAI $=$ Separation Anxiety Inventory, Child and Parent version 
Analysis of the SAI-P showed the same pattern of results, with significant Time, $F(1,26)=39.76, p<0.001$, and Group, $F(1,26)=25.56, p<0.001$, main effects and Time $\times$ Group interaction, $F(1,26)=18.99, p<0.001$.

\section{Vigilance and Avoidance in Children's Gaze After CBT}

The procedure to analyze pre-treatment bias scores was comparable to the analysis in the In-Albon et al. (2010) study. The pre-treatment bias scores were calculated as the percent of time the child spent looking at separating pictures as a function of total time he or she spent looking at either the separating or the reuniting pictures. The means of these bias scores by each child group over the $4 \mathrm{~s}$ of viewing time, subdivided into eight $500 \mathrm{~ms}$ intervals, are presented in Table 2 and graphically in Fig. 1.

We conducted a 2 (child group: SAD and non-anxious) $\times 2$ (pre-post: pre- and post-treatment) $\times 8$ (time interval, ending at: $500 \mathrm{~ms}, 1,000 \mathrm{~ms}, 1,500 \mathrm{~ms}, 2,000 \mathrm{~ms}, 2,500 \mathrm{~ms}$, $3,000 \mathrm{~ms}, 3,500 \mathrm{~ms}, 4,000 \mathrm{~ms}$ ) mixed ANOVA with prepost and time interval as within-subjects factors and child group as between-subjects factor. Results yielded a significant three-way interaction, $F(3.3,81.8)=6.77, p<0.001, \eta_{\mathrm{p}}^{2}=$ 0.21 (see Table 3). From visual inspection of Fig. 1, it is evident that the four groups differ regarding their time trajectories: children with SAD at pre-treatment show the vigilance and avoidance pattern with a vigilance peak at 1,500 2,000 ms following stimulus onset consistent to the trajectory at pre-treatment. In contrast, to the other three trajectories in Fig. 1 show no vigilance effect for children with SAD at posttreatment and non-anxious children at both pre- and post assessments. Indeed, separate analyses for pre and post assessments revealed a significant two-way interaction for group $\times$ time interval, $F(4.0,104.4)=4.23, p=0.003, \eta_{\mathrm{p}}^{2}=$ 0.14 , at pre assessment but not at post assessment, $F(3.8$, $96.1)=1.73, p=0.15, \eta_{\mathrm{p}}^{2}=0.065$. This indicates that existing differences between SAD and non-anxious children at pre assessment no longer existed post assessment, suggesting that the treatment for children with SAD was successful.

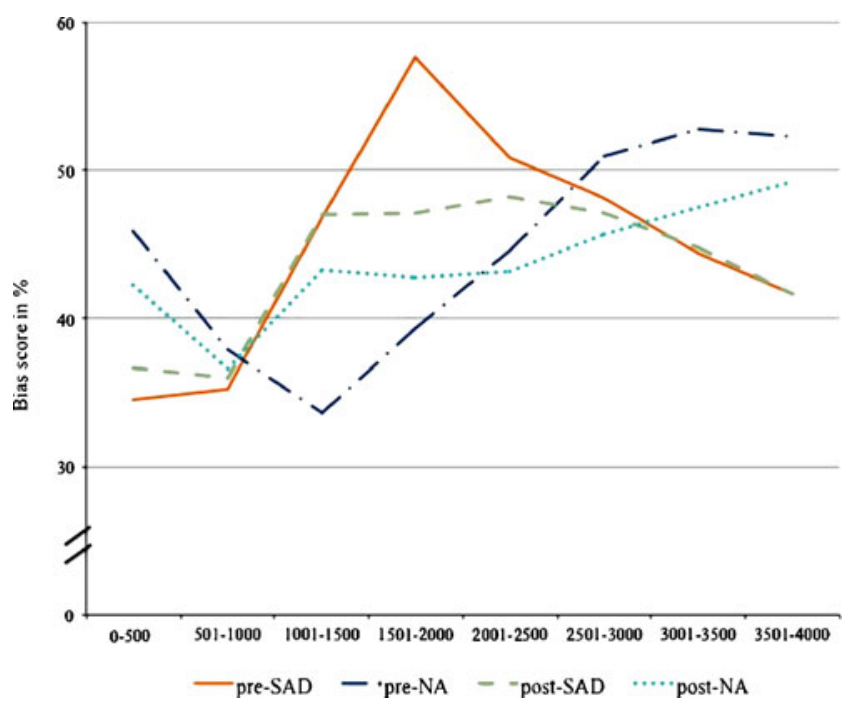

Fig. 1 Bias scores showing the percentages of time spent looking at separating pictures as a function of total time gazing at both pictures at each $500 \mathrm{~ms}$ interval for the two child groups (children with SAD and non-anxious (NA)) children pre- and post-treatment

Results also yielded significant two-way interactions for group $\times$ time interval, $F(3.9,98.2)=3.19, p=0.017, \eta_{\mathrm{p}}^{2}=$ 0.11 , and for pre-post $\times$ time interval interaction, $F(3.3$, $81.8)=4.90, p=0.003, \eta_{\mathrm{p}}^{2}=0.16$, and a significant main effect for time interval, $F(3.9,98.2)=2.50, p=0.048, \eta_{\mathrm{p}}^{2}=$ 0.09 (see Table 3). However, these will not be discussed in the light of the observed three-way interaction. The covariates gender and age had no influence on the effect of the planned factors on each of the outcomes analyzed.

\section{Discussion}

The present study has shown that the vigilance-avoidance viewing behavior changed after $\mathrm{CBT}$ in children with SAD. After CBT, the viewing behavior of children with SAD changed so that the vigilance effect was no longer visible. The results are in line with findings from adult studies

Table 2 Bias scores pre- and post-treatment by child anxiety group at each $500 \mathrm{~ms}$ interval

Time interval since onset of slide

\begin{tabular}{lllllllll}
\hline $\begin{array}{l}\text { Child group } \\
\text { Bias score pre-treatment }\end{array}$ & $0-500 \mathrm{~ms}$ & $501-1000 \mathrm{~ms}$ & $1001-1500 \mathrm{~ms}$ & $1501-2000 \mathrm{~ms}$ & $2001-2500 \mathrm{~ms}$ & $2501-3000 \mathrm{~ms}$ & $3001-3500 \mathrm{~ms}$ & $3501-4000 \mathrm{~ms}$ \\
$\begin{array}{l}\text { SAD } \\
37.08\end{array}$ & 35.4 & 47.44 & 57.21 & 49.41 & 48.31 & 43.73 & 43.06 \\
$\begin{array}{l}\text { Nonanxious } \\
\text { children }\end{array}$ & 45.83 & 37.88 & 33.65 & 39.31 & 44.53 & 50.97 & 52.73 & 52.23 \\
$\begin{array}{l}\text { Post-treatment } \\
\text { SAD }\end{array}$ & & & & & & & \\
$\begin{array}{l}\text { Nonanxious } \\
\text { children }\end{array}$ & 46.69 & 36.00 & 47.04 & 47.1 & 48.22 & 47.09 & 44.72 & 41.63 \\
\hline
\end{tabular}


Table 3 Mixed analysis of variance including the predictors child group (2 levels: SAD, non-anxious), pre-post treatment (2 levels), and time interval ( 8 levels)

\begin{tabular}{llllll}
\hline & SSQ & df & $F$ & $p$ & $\eta_{\mathrm{p}}^{2}$ \\
\hline Between subjects & & & & & \\
Group & 75.4 & 1 & 0.08 & 0.78 & 0.003 \\
Rest & 23976.0 & 25 & - & - & - \\
Within subjects: pre-post & & & & & \\
Pre-post & 31.2 & 1 & 0.83 & 0.37 & 0.032 \\
Pre-post $\times$ Group & 81.6 & 1 & 2.18 & 0.153 & 0.080 \\
Rest & 936.7 & 25 & - & - & - \\
Within subjects: interval & & & & & \\
Interval & 5564.6 & $3.9^{\mathrm{a}}$ & 2.50 & 0.048 & 0.091 \\
Interval $\times$ Group & 7098.6 & $3.9^{\mathrm{a}}$ & 3.19 & 0.017 & 0.113 \\
Rest & 55572.9 & $98.2^{\mathrm{a}}$ & & & \\
Within subjects: pre-post $\times$ interval & & & & \\
Pre-post $\times$ interval & 765.1 & $3.3^{\mathrm{a}}$ & 4.90 & 0.003 & 1.64 \\
Pre-post $\times$ interval $\times$ group & 1056.7 & $3.3^{\mathrm{a}}$ & 6.77 & $<0.001$ & 0.213 \\
Rest & 3904.4 & 81.8 & & & \\
\hline
\end{tabular}

${ }^{a}$ Values adjusted according to Greenhouse-Geisser

indicating that attentional bias is not an unalterable phenomenon (e.g., Mathews et al. 1995; Mogg et al. 1995). The present study adds to current understanding that changes in the viewing behavior can be demonstrated using a continuous measure of attention, such as eye tracking methodology. Our results differ from the study by Waters et al. (2008), which indicated that children with different anxiety disorders following CBT continued to show a significant attentional bias towards threat on a visual-probe task. The differences may result from the fact that the current study specifically investigated children with SAD using disorder specific material. Waters et al. (2008) pointed out that the broad-based pictorial stimuli utilized in their study were insensitive to changes. As mentioned before, in the present study attentional bias was assessed using eye tracking as a continuous measure of attention. This methodology also differs from the studies by Legerstee et al. $(2009,2010)$ using a pictorial dot-probe task at pre- and post-treatment. Legerstee et al. (2010) demonstrated that changes in selective attention to severely threatening pictures were significantly associated with treatment success. Treatment responders showed a reduction of their predisposition to selectively attend away or toward severely threatening pictures. However, nonresponders did not show any changes in selective attention due to CBT. Similarly, Legerstee et al. (2009) showed that selective attention has been a predictor of treatment success in childhood anxiety disorders. Using also a dot-probe task, responders of the CBT treatment showed a selective attention away from severe threat. Non-responders, on the other hand, showed still attention toward threat.
To our knowledge ours is the first study to investigate viewing behavior using eye tracking methodology at preand post-treatment. It is important to note that the viewing behavior of non-anxious children did not significantly differ between the two assessments, indicating reliability of the procedure.

Although the present study extended previous research, the current findings need to be considered in light of some limitations. The vigilance avoidance pattern was not reassessed at follow-up intervals after treatment. Furthermore, an additional investigation of a clinical waitlist group would have shown that changes in the viewing behavior were in fact due to treatment rather than caused by natural remission over time. Because there was "only" one child still meeting SAD criteria after treatment, group differences between responders and non-responders could not be investigated. However, we could show that in the non-anxious group there was no change in viewing pattern between the two assessments. It is a limitation of current research in general, that the role of the models regarding causality cannot be answered based on current empirical studies, which are mostly cross-sectional. However, our study could show that vigilance avoidance pattern is 1 ) associated with a diagnosis of SAD and 2) can be changed by CBT. Using Kraemer's definition of a causal risk factor, these results provide first but of course not sufficient indication, that attentional bias may qualify as a risk or even causal risk factor for anxiety disorders in children (Kraemer et al. 1997). Factors such as genetic, temperamental, learning and intergenerational transmission are in discussion regarding the origin of information processing biases (see Hadwin and Field 2010 for an overview). Future research should also investigate the mechanisms maintaining anxiety as well as the components that change attentional bias. Is change induced by exposure, as suggested by Lavy et al. (1993), thereby leading to new structures through activation and modifying the fear network (see also Foa and Kozak 1986) or by other components (e.g. cognitive restructuring)? For example, Legerstee et al. (2010) showed that exposure interventions were efficacious for children who did not engage their attention toward severe threat. Children having difficulties in disengaging their attention away from threat (showing vigilance), however, did not benefit from exposure interventions. The authors suggest that these children would probably benefit more from learning to redirect their attention away from threat to neutral or pleasant aspects of a situation. In addition, future studies should examine attention training (cognitive bias modification) in children with anxiety disorders and measure whether it is as efficacious in children as it is in adults with anxiety disorders (see Journal of Abnormal Psychology, Special Issue 2009, 118 (1); Cowart and Ollendick 2011; Hakamata et al. 2010). 
In conclusion, the present study demonstrated that the vigilance-avoidance pattern of children with SAD assessed with eye tracking methodology could be modified through CBT. Following treatment, the vigilance pattern of children with SAD reduced significantly.

Acknowledgments This study was supported by grant project PP001-68701; 105311-116517/1) awarded to Silvia Schneider by the Swiss National Science Foundation. We appreciate the participants in this study, as well as the research assistants and graduate students on the project at the University of Basel for their assistance in data collection and management.

\section{References}

Allen, J. L., Lavallee, K. L., Herren, C., Ruhe, K., \& Schneider, S. (2010). DSM-IV criteria for childhood separation anxiety disorder: informant, age, and sex differences. Journal of Anxiety Disorders, 24(8), 946-952.

American Psychiatric Association. (2000). Diagnostic and Statistical Manual of Mental Disorders: 4th edition text revision. Washington DC: American Psychiatric Press.

Beck, A. T., Emery, G., \& Greenberg, R. L. (1985). Anxiety disorder and phobias: A cognitive perspective. New York: Basic Books.

Bögels, S., \& Mansell, W. (2004). Attention processes in the maintenance and treatment of social phobia: hypervigilance, avoidance and selffocused attention. Clinical Psychology Review, 24, 827-856.

Brückl, T. M., Wittchen, H.-U., Höfler, M., Pfister, H., Schneider, S., \& Lieb, R. (2007). Childhood separation anxiety and the risk for subsequent psychopathology: results from a community study. Psychotherapy and Psychosomatics, 76, 47-56.

Cartwright-Hatton, S., McNicol, K., \& Doubleday, E. (2006). Anxiety in a neglected population: prevalence of anxiety disorders in preadolescent children. Clinical Psychology Review, 26, 817-833.

Cisler, J. M., \& Koster, E. H. W. (2010). Mechanisms of attentional biases towards threat in anxiety disorders: an integrative review. Clinical Psychology Review, 30, 203-216.

Cowart, M. J. \& Ollendick, T. H. (2011). Attention training in socially anxious children: a multiple baseline design analysis. Journal of Anxiety Disorders, 25 (7), 972-977.

Field, A. P. (2006). Watch out for the beast: fear information and attentional bias in children. Journal of Clinical Child and Adolescent Psychology, 35, 431-439.

Foa, E. B., \& Kozak, M. J. (1986). Emotional processing of fear: exposure to corrective information. Psychological Bulletin, 99, $20-35$.

Gamble, A. L., \& Rapee, R. M. (2009). The time-course of attentional bias in anxious children and adolescents. Journal of Anxiety Disorders, 23, 841-847.

Garner, M., Mogg, K., \& Bradley, B. P. (2006). Orienting and maintenance of gaze to facial expressions in social anxiety. Journal of Abnormal Psychology, 115, 760-770.

Hadwin, J. A., \& Field, A. P. (2010). Information processing biases in child and adolescent anxiety. Chichester: Wiley.

Hakamata, Y., Lissek, S., Bar-Haim, Y., Britton, J. C., Fox, N. A., Leibenluft, E., Ernst, M., \& Pine, D. S. (2010). Attention bias modification treatment: a meta-analysis toward the establishment of novel treatment for anxiety. Biological Psychiatry, 68, 982-990.

Heinrichs, N., \& Reinhold, N. (2010). Experimentelle Augenbewegungsmessungen bei Kindern und Jugendlichen - Relevanz für psychopathologische Prozesse am Beispiel der Sozialen Angst. Kindheit und Entwicklung, 19, 12-20.
In-Albon, T., \& Schneider, S. (2007). Psychotherapy of childhood anxiety disorders: a meta-analysis. Psychotherapy and Psychosomatics, 76, 15-24.

In-Albon, T., Kossowsky, J., \& Schneider, S. (2010). Vigilance and avoidance in children with separation anxiety disorder using an eye movement paradigm. Journal of Abnormal Child Psychology, $38,225-235$.

In-Albon, T., Dubi, K., Adornetto, C., Blatter-Meunier, J., \& Schneider, S. (2011). Neue Ansätze in der Diagnostik von Angststörungen im Kindes- und Jugendalter. Klinische Diagnostik und Evaluation, 4, 133-147.

Kessler, R. C., Berglund, P., Demler, O., Jin, R., Merikangas, K. R., \& Walters, E. E. (2005). Lifetime prevalence and age-of-onset distributions of DSM-IV disorders in the National Comorbidity Survey Replication. Archives of General Psychiatry, 62, 593-603.

Kraemer, H. C., Kazdin, A. E., Offord, D. R., Kessler, R. C., Jensen, P. S. \& Kupfer, D. J. (1997). Coming to terms with the terms of risk. Archives of General Psychiatry, 54, 337-343.

Lavy, E., van den Hout, M., \& Arntz, A. (1993). Attentional bias and spider phobia: conceptual and clinical issues. Behaviour Research and Therapy, 31, 17-24.

Legerstee, J. S., Tulen, J. H. M., Kallen, V. L., Dieleman, G. C., Treffers, P. D. A., Verhulst, F. C., \& Utens, E. M. W. J. (2009). Threat-related selective attention predicts treatment success in childhood anxiety disorders. Journal of the American Academy of Child and Adolescent Psychiatry, 48, 196-205.

Legerstee, J. S., Tulen, J. H. M., Dierckx, B., Treffers, P. D. A., Verhulst, F. C., \& Utens, E. M. W. J. (2010). CBT for childhood anxiety disorders: differential changes in selective attention between treatment responders and non-responders. Journal of Child Psychology and Psychiatry, 51, 162-172.

Lewinsohn, P. M., Holm-Denoma, J. M., Small, J. W., Seeley, J. R., \& Joiner, T. E. (2008). Separation anxiety disorder in childhood as a risk factor for future mental illness. Journal of the American Academy of Child and Adolescent Psychiatry, 47, 549-556.

MacLeod, C., Rutherford, E., Campbell, L., Ebsworthy, G., \& Holker, L. (2002). Selective attention and emotional vulnerability: assessing the causal basis of their association through the experimental manipulation of attentional bias. Journal of Abnormal Psychology, 111, 107-123.

Mathews, A., \& Mackintosh, B. (1998). A cognitive model of selective processing in anxiety. Cognitive Therapy and Research, 22, 539560.

Mathews, A., Mogg, K., Kentish, J., \& Eysenck, M. (1995). Effects of psychological treatment on cognitive bias in generalized anxiety disorder. Behaviour Research and Therapy, 33, 293-303.

Mattia, J. I., Heimberg, R. G., \& Hope, D. A. (1993). The revised Stroop color-naming task in social phobics. Behaviour Research and Therapy, 31, 305-314.

Mogg, K., \& Bradley, B. P. (1998). A cognitive-motivational analysis of anxiety. Behaviour Research and Therapy, 36, 809-848.

Mogg, K., Bradley, B. P., Millar, N., \& White, N. (1995). A follow-up study of cognitive bias in generalized anxiety disorder. Behaviour Research and Therapy, 33, 927-935.

Puliafico, A. C., \& Kendall, P. C. (2006). Threat-related attentional bias in anxious youth: a review. Clinical Child and Family Psychology Review, 9, 162-180.

Rinck, M., \& Becker, E. S. (2006). Spider fearfuls attend to threat, then quickly avoid it: evidence from eye movements. Journal of $A b$ normal Psychology, 115, 231-238.

Schneider, S., \& In-Albon, T. (2005). Separation Anxiety Inventory, child and parent version. Unpublished manual, University of Basel.

Schneider, S., \& Lavallee, K. L. (2012). Family-based cognitivebehavioral treatment for separation anxiety disorder. In C. A. Essau \& T. Ollendick (Eds.), Treatment of childhood and adolescent anxiety. Hoboken: Wiley-Blackwell. 
Schneider, S., Unnewehr, S., \& Margraf, J. (2009a). Diagnostisches Interview psychischer Störungen im Kindes- und Jugendalter (Kinder-DIPS). Berlin: Springer.

Schneider, S., Suppiger, A., Adornetto, C., \& Unnewehr, S. (2009b). Diagnostisches Interview bei psychischen Störungen im Kindesund Jugendalter (Kinder-DIPS), Handbuch. Heidelberg: Springer.

Schneider, S., Blatter, J., Herren, C., Adornetto, C., In-Albon, T., \& Lavallee, K. (2011). Disorder-specific cognitive-behavioral treatment for separation anxiety disorder in young children: a randomized waitlist-controlled trial. Psychotherapy and Psychosomatics, $80,206-215$
Shear, K., Jin, R., Ruscio, A. M., Walters, E. E., \& Kessler, R. C. (2006). Prevalence and correlates of estimated DSM-IV child and adult separation anxiety disorder in the national comorbidity survey replication. The American Journal of Psychiatry, 163, 1074-1083.

Tobii Technology AB. (http://www.tobii.com).

Waters, A. M., Wharton, T. A., Zimmer-Gembeck, M. J., \& Craske, M. G. (2008). Threat- based cognitive biases in anxious children: comparison with non-anxious children before and after cognitive behavioural treatment. Behaviour Research and Therapy, 46, 358-374. 